\title{
Generalizing Effects of Frontostriatal Structural Connectivity on Self-Esteem Using Predictive Modeling
}

\author{
Robert S. Chavez, Dale T. Tovar, Moriah S. Stendel, and Taylor D. Guthrie
}

Department of Psychology, University of Oregon, Eugene, Oregon, USA

\begin{abstract}
Determining the generalizability of biological mechanisms supporting psychological constructs is a central goal of cognitive neuroscience. Self-esteem is a popular psychological construct that is associated with a variety of measures of mental health and life satisfaction. Recently, there has been interest in identifying biological mechanisms that support individual differences in self-esteem. Understanding the biological basis of self-esteem requires identifying predictive biomarkers of self-esteem that generalize across groups of individuals. Previous research using diffusion magnetic resonance imaging has shown that selfesteem is related to the integrity of structural connections linking frontostriatal brain systems involved in self-referential processing and reward. However, these findings were based on a small, relatively homogeneous group of participants. In the current study, we used an out-of-sample predictive modeling approach to generalize the results of the previous study to an independent sample of participants more than twice the size of the original study. We found that both linear univariate and multivariate machine learning models trained on frontostriatal integrity from the original data significantly predicted self-esteem in the independent dataset. These findings underscore the relationship between self-esteem and frontostriatal connectivity and suggest these results are robust to differences in scanning acquisition, analytic methods, and participant demographics.
\end{abstract}

self-esteem | predictive modeling | frontostriatal connectivity | social neuroscience

Correspondence: rchavez@uoregon.edu

NOTE: The current manuscript is a preprint and may not perfectly reflect the final article.

Humans are motivated to engage in positive self-evaluation and typically hold favorable views of the self. Variability in these propensities contribute to an individual's sense of self-esteem and are related to a number of symptoms of psychological disorders such as those in depression and anxiety (Sowislo \& Orth, 2013) as well as a variety of life satisfaction and well-being measures (Orth et al., 2012). A person's sense of self-esteem relies on the ability to integrate evaluative processing with information about the self, and this has been the focus of empirical work on the biological basis of self-esteem. However, establishing a biological basis of selfesteem requires identifying markers of self-esteem that are generalizable across groups of people and experimental contexts.

Across dozens of studies using different experimental paradigms, researchers have demonstrated that medial pre- frontal cortex (MPFC) exhibits robust activation during selfreferential processing (Denny et al., 2012; Wagner et al., 2012). Frontocortical systems are thought to integrate information across different cortical areas and with subcortical structures (Miller \& Cohen, 2001). The MPFC also has known efferent connectivity to areas within the ventral striatum (Groenewegen et al., 1999; Sesack et al., 1989) which are involved in motivation and reward-related processes (Knutson et al., 2001). Indeed, self-esteem is hypothesized to reflect the degree to which a person is able to integrate positive evaluation with information about the self. Though encompassing very different lines of research, these previous studies have led researchers to posit that individual differences in self-esteem may be related to the structural integrity of frontostriatal connections linking the MPFC to the ventral striatum.

Diffusion magnetic resonance imaging (dMRI) is a neuroimaging method for assaying microstructural properties of white matter tracts that connect brain regions together. In a previous study using dMRI, researchers found a positive correlation between measures of frontostriatal white matter integrity and trait self-esteem (Chavez \& Heatherton, 2015). Additionally, in a follow up study using the same participants, they found that frontostriatal integrity of these pathways correlated with the change in self-esteem over participants' first year of college several months after initial scanning (Chavez \& Heatherton, 2017). However, both of these investigations relied on a relatively small sample of college students and were collected using dMRI acquisition protocols that have since been superseded (Graham et al., 2016). Thus, it remains an open question whether or not these results could generalize to an independent sample of participants using more sophisticated data analytic methods.

Moreover, neuroimaging studies have increasingly deployed multivariate methods, such as multivoxel pattern analysis (Haxby et al., 2014; Weaverdyck et al., 2020), to capture information about brain activity and structure that is distributed across different parts of the brain. These methods have also been increasingly applied to topics in social cognition and self-representation (Wagner et al., 2019). Indeed, multivariate prediction models may be among the most powerful and direct ways to develop, test, and share biomarkers of psychological phenomena using neuroimaging because of their success in predicting a variety of mental states, mental health status, and individual differences variables (Woo et al., 
2017). As such, utilizing a multivariate approach for training and testing a predictive modeling of self-esteem is also warranted.

In the current study, we sought to directly evaluate the predictive utility of these original findings using an out-ofsample predictive modeling approach. Using the frontostriatal white matter tracts identified in a previous study, we trained a univariate linear regression model to predict selfesteem using average frontostriatal tract integrity $(\mathrm{N}=48)$. Furthermore, to capture information distributed across voxel patterns, we also trained multivariate ridge regression models both within the frontostriatal tracts and across the remaining white matter voxels in the rest of the brain. Next, we tested these models on a newly collected sample of participants $(\mathrm{N}=109)$ with updated acquisition and analysis protocols and in a different scanner than the original study. This predictive modeling framework provides a direct, model-based method for evaluating the replicability and generalizability of the original findings.

\section{Methods}

We used a predictive modeling framework to directly test the generalizability of the previous findings from Chavez and Heatherton (2015) to a newly collected, completely out-ofsample dataset. To do this, we used measures of white matter integrity along the tracts identified in the original study as model training data for fitting both univariate and multivariate predictive models. These models were then directly applied to the newly collected data to generate model-based predictions of self-esteem based on frontostriatal white matter integrity. These predictions were then tested against the measured self-esteem scores in the new sample of participants. Both samples used the same measure of self-esteem, but there are some differences in the dMRI acquisition and preprocessing procedures. Additional details of the experimental procedure are described below.

\section{Original Sample.}

Participants. Forty-eight volunteers between the ages of 18 and 19 (28 female) were recruited from the Dartmouth College community. All participants completed behavioral questionnaires and high-resolution anatomical and dMRI scans. All participants were screened to be right-handed and selfreported no current or history of psychiatric or neurological conditions. Participants gave informed consent in accordance with the guidelines set by the Committee for the Protection of Human Subjects at Dartmouth College and received course credit or were paid for their participation.

Self-Esteem Measure. Each participant's self-esteem was assessed outside the scanner using the Janis \& Field Feelings of Inadequacy Scale (Fleming \& Courtney, 1984), a thirtysix item inventory of global self-esteem shown to have robust psychometric properties (Blascovich \& Tomaka, 1991). We asked participants to report general self-evaluative feelings over the course of the previous year. This measure was used for both the original and the new samples of participants.
Image Acquisition. Magnetic resonance imaging was conducted with a Philips Achieva 3.0 Tesla scanner using a thirty-two-channel phased array coil. Structural images were acquired using a T1-weighted MP-RAGE protocol (220 sagittal slices; TR: $8.176 \mathrm{~ms}$; TE: $3.72 \mathrm{~ms}$; flip angle: $8^{\circ}$; $1 \mathrm{~mm}$ isotropic voxels). Diffusion weighted images were collected using 70 contiguous 2-mm-thick axial slices with 32 diffusion directions (91ms TE, 8848 TR, $1000 \mathrm{~s} / \mathrm{mm} 2 \mathrm{~b}-$ value, $240 \mathrm{~mm}$ FOV, $90^{\circ}$ flip angle, $1.875 \times 1.875 \times 2 \mathrm{~mm}$ voxel size). Two diffusion scans were acquired and averaged per subject.

Preprocessing. All dMRI data were analyzed using the Diffusion Toolbox in FSL (Behrens et al., 2003). Preprocessing included brain extraction, motion correction, and distortion correction using the 'eddycorrect' tool. We used probabilistic tractography to delineate white matter tracts between the MPFC and bilateral ventral striatum defined anatomically using an automated subcortical segmentation tool, FIRST (Patenaude et al., 2011). A dual-fiber model was implemented with BEDPOSTx (Behrens et al., 2007) to account for crossing fiber uncertainty in the diffusion imaging signal. Using two-mask seeding, 5000 probabilistic tract-streamlines were taken at each voxel. This method allowed resulting tractography maps to only include streamlines passing through both seed masks. These results were then normalized to Montreal Neurological Institute (MNI) standard space using nonlinear registration using FSL's FNIRT tool. To ensure tracts were consistent among participants, registered tractography results were then binarized and overlaid with all other participants and thresholding was set at the group level such that there was a $50 \%$ tract probability across participants in standard space. In this analysis, we quantified individual differences in white matter integrity using fractional anisotropy (FA), a measure of directional coherence reflecting a combination general microstructural properties of white matter such as myelination and axonal fidelity (Song et al., 2002). These values were then used for the structural connectivity correlational analyses with the self-esteem measures.

\section{New Sample.}

Participants. One-hundred and nine volunteers between the ages of 18 and $51(\mathrm{M}=23.15 ; \mathrm{SD}=6.81 ; 41$ female $)$ were recruited from the University of Oregon and local Eugene community as part of a larger neuroimaging study on social cognition. Additional recruitment screening procedures were the same as the original sample. Participants gave informed consent in accordance with the guidelines set by the Internal Review Board at University of Oregon and all were paid for their participation.

Image Acquisition. Magnetic resonance imaging was conducted with a Siemens Skyra 3.0 Tesla scanner using a thirtytwo-channel head coil. Structural images were acquired using a T1-weighted MP-RAGE protocol (220 sagittal slices; TR: $2.5 \mathrm{~ms}$; TE: $3.43 \mathrm{~ms}$; flip angle: $7^{\circ} ; 1 \mathrm{~mm}$ isotropic voxels). Diffusion weighted images were collected using 70 
contiguous 2-mm-thick axial slices with 60 diffusion directions (75.4 ms TE , $3.92 \mathrm{TR}, 1000 \mathrm{~s} / \mathrm{mm} 2 \mathrm{~b}$-value, $90^{\circ}$ flip angle, $2 \mathrm{~mm}$ isotropic voxel size.

Preprocessing. The preprocessing of the new sample largely followed the original but deviated from it where advancements in acquisition allowed for a better-quality image reconstruction. Specifically, in addition to standard eddy current and motion correction, we applied FSL's 'topup' and 'eddy' tools which use reversed phase-encode blips, resulting in pairs of images with distortions going in opposite directions which more accurately estimates and corrects for susceptibility induced distortions (Graham et al., 2016). These tools superseded the eddycorrect tool used in the original sample and are used here to test predictive robustness against discrepancies in data analytic procedures. Data were registered to the MNI template in the same manner as the original data. Frontostriatal tracts delineated in the original sample were overlaid on each participant's FA image for use in the univariate and multivariate model evaluation.

\section{Predictive Modeling Procedures.}

We used both univariate and multivariate modeling methods to predict self-esteem from FA values in the training data (i.e.the original sample). The frontostriatal regions of interest used in both the univariate and multivariate analyses were taken directly from the tractography results in Chavez and Heatherton (2015). For the univariate analyses, we calculated the mean FA across all voxels within the bilateral frontostriatal tracts. To ensure that masks only included white matter, we only included values with a minimum of $\mathrm{FA}=0.2$ (Smith et al., 2006). These values were then used as predictors to fit a simple linear regression model of self-esteem scores. This model was then applied to the FA values from the testing data (i.e. the new sample) to generate a predicted self-esteem score. We henceforth refer to this model as the univariate frontostriatal model.

Rather than averaging values across voxels as in the univariate analyses, multivariate methods aim to capture information that is distributed across voxel patterns and can be fit using standard machine learning algorithms (Woo et al., 2017). Here, we fit a voxelwise multivariate model of self-esteem within the same frontostriatal tracts from the univariate model using the nltools package in Python (https://github.com/cosanlab/nltools) which leverages neuroimaging tools from the nilearn package (Abraham et al., 2014) as well as machine learning algorithms implemented in scikit-learn (Pedregosa et al., 2011). Within the training dataset, we fit the model using a ridge regression model, alpha $=100$ within a $\mathrm{k}=10$-fold cross validation scheme. The results of this procedure generated a weight map based on the voxelwise parameters of the model. Weight maps reflect the direction and degree to which each voxel contributed to the predicted self-esteem value and can be used to evaluate the spatial location of the most informative voxels in the model. We then added the model intercept to the dot product of the weight map to generate the model-generated predicted self- esteem score for each participant. These predicted values were then compared against the actual measured self-esteem values within each participant. We henceforth refer to this model as the multivariate frontostriatal model.

Finally, to test the specificity of the multivariate model to frontostriatal circuits, we also fit an FA model to predict selfesteem based on remaining white matter voxels for the rest of the brain outside of the frontostriatal tracts. For the nonfrontostriatal voxels, we restricted these analyses to white matter voxels that showed a high subject-wise signal-to-noise (SNR) ratio based on the ratio of the mean FA to the standard deviation across subjects. We then thresholded SNR at a value of 5, such that the mean FA across subjects was at least 5 times larger than the standard deviation, and binarized the results to generate a mask. The resulting mask from this procedure included the bulk of prominent white matter regions across the cortex, subcortex, and cerebellum. The final non-frontostriatal mask was then generated by subtracting the binarized frontostriatal tracts from the SNR mask. Other than the white matter areas examined, the model fitting procedures followed the multivariate frontostriatal model exactly. We henceforth refer to this as the non-frontostriatal model

\section{Results}

\section{Within-Sample Statistics.}

Self-esteem scores within the original sample had $M=113$, $\mathrm{SD}=11.2$, and $\mathrm{M}=90.3, \mathrm{SD}=19.8$ for the new sample. These results were significantly different $\mathrm{t}(155)=7.42$, $\mathrm{p}<.001$, indicating that the sample of participants in the new sample had lower self-esteem on average than those in the original sample. Frontostriatal tracts identified in the original analysis are displayed in Figure 1A and were used in both the model training and testing samples. Within the training data, self-esteem and frontostriatal integrity showed relatively strong, significant correlations of $\mathrm{R}(46)=.50, \mathrm{p}<.001$ and $\mathrm{r}(46)=.44, \mathrm{p}$ $<.001$ in the right and left hemispheres respectively. Within the testing data, the raw correlations of self-esteem and frontostriatal integrity were $\mathrm{R}(107)=.2, \mathrm{p}=.036$ and $\mathrm{r}(107)=$ $.17, \mathrm{p}=.07$ in the right and left hemispheres respectively. These results show that the raw correlation values mirror the pattern of results from the original study, with a stronger relationship in the right hemisphere compared to the left hemisphere. Consistent with previous replication studies (Patil et al., 2016), these relationships were attenuated relative to the original study.

\section{Out-of-Sample Model Performance.}

The central goal of this study was to directly test the generalizability of the findings from the original study using a predictive modeling framework. Using the linear univariate model, the multivariate frontostriatal model, and the multivariate non-frontostriatal model, we fit the parameters estimated from the data in the original study before applying them to the newly collected data. This procedure generates a predicted value of self-esteem based on each model for each participant in the testing data. These predicted values can 
A.

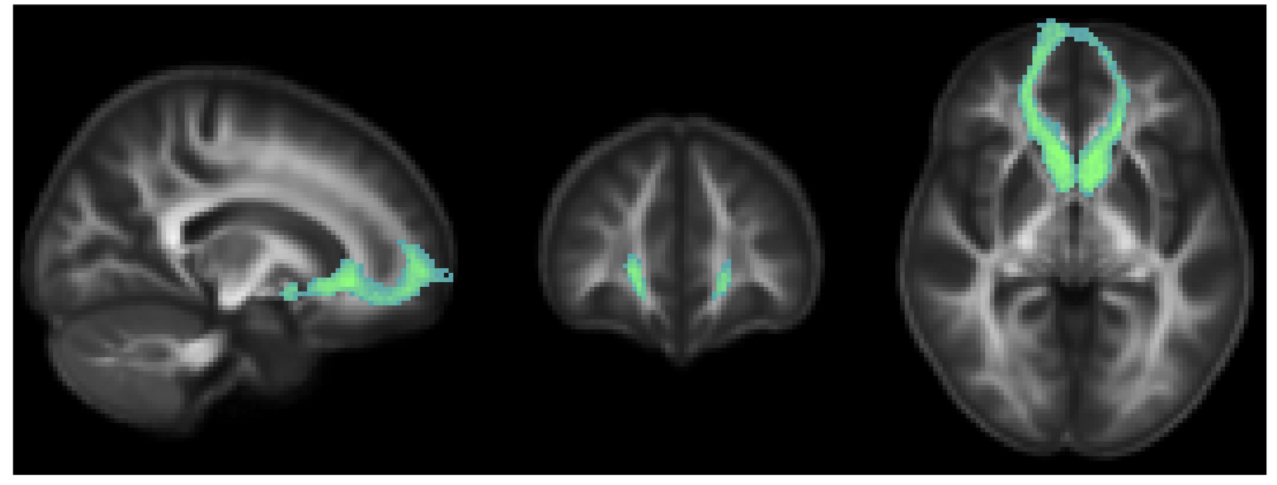

B.
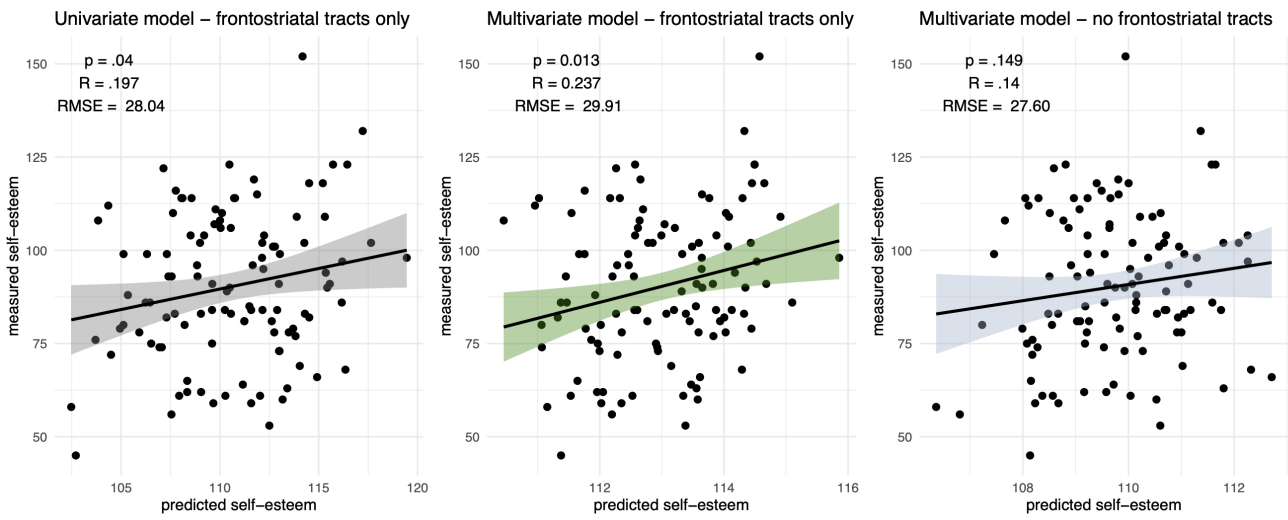

Fig. 1. Frontostriatal white matter tracts and the relationship between the participant's self-esteem scores and model predictions. (a) Frontostriatal tracts identified using probabilistic tractography procedures from Chavez \& Heatherton (2015). These tracts were used as the region of interest for both the univariate and multivariate frontostriatal model training in the current study. (b) Scatterplots depicting the relationship between predicted self-esteem based on each model and actual self-esteem measured within each participant. All model predictions were positively with measured self-esteem scores. However, only the frontostriatal models were significantly related in the out-ofsample data.

then be evaluated against the actual self-esteem scores measured in each participant.

Scatter plots depicting the relationship between the predicted and measured self-esteem scores for each model are shown in Figure 1B. For the univariate model, we found a significant positive relationship between the self-esteem model predictions and the empirically measured self-esteem scores, $\mathrm{R}(107)=.2, \mathrm{p}=.04, \mathrm{RMSE}=28.04$. Specifically, the higher the predicted value of self-esteem was from the model, the higher the measured self-esteem score tended to be in the newly collected data.

The results of the multivariate frontostriatal model also showed a significant positive relationship between predicted and measured self-esteem scores, $\mathrm{R}(107)=.237, \mathrm{p}=.013$, RMSE $=29.910$. Weight maps for both the frontostriatal tracts and the remainder of the white matter not including the frontostriatal tracts are displayed in Figure 2. The results from the multivariate non-frontostriatal model were in the same direction as the other models, but did not reach statistical significance $\mathrm{R}(107)=.139, \mathrm{p}=.149, \mathrm{RMSE}=27.6$. This analysis provides evidence that these relationships are specific to the frontostriatal tracts, and not a result of simply applying multivariate methods more generally.

Finally, in the testing data, there was a participant with a self-esteem score well above the mean. To ensure that our results are robust to this outlier, we performed the analysis again after excluding this participant. Here found that our multivariate frontostriatal tracts-only model remained significant ( $\mathrm{p}=.03, \mathrm{R}=.2$, RMSE 29.81) and our multivariate nonfrontostriatal tracts model remain non-significant $(\mathrm{p}=.15, \mathrm{R}$ $=.14, \mathrm{RMSE}=27.428$ ). These results are consistent with our original findings and thus do not change the interpretation of our findings. Code and plots for these analyses are included in the OSF repository.

\section{Discussion}

Previous research has shown that the integrity of frontostriatal white matter tracts linking brain regions involved in selfreferential processing and reward was positively associated with self-esteem (Chavez and Heatherton, 2015). In the current study, we used a predictive modeling framework to directly test the generalizability of the findings from these previous findings to a sample of subjects collected in different scanner with updated acquisition methods. Using the previous study to train univariate and multivariate predictive models, we tested the predictive accuracy of these models in a larger, newly collected sample of participants. We found that both univariate and multivariate frontostriatal models significantly predicted self-esteem in the new sample. Furthermore, a multivariate model using non-frontostriatal white matter did not significantly predict self-esteem in the new sample, suggesting that these findings may be specific to frontostriatal 
A.

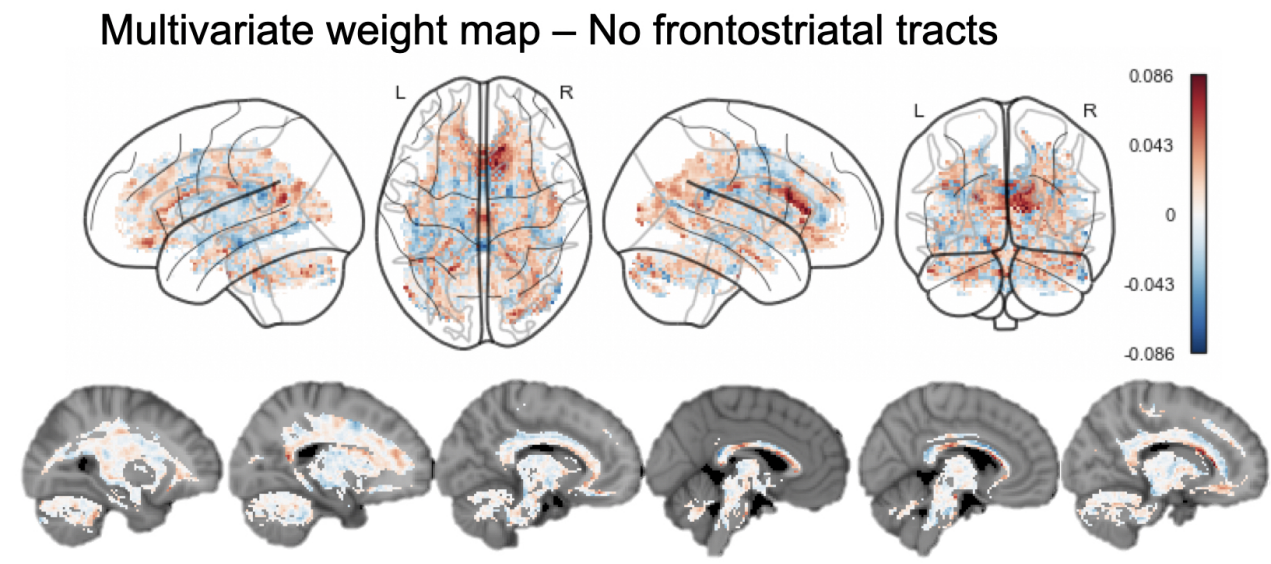

B. Multivariate weight map - Frontostriatal tracts only

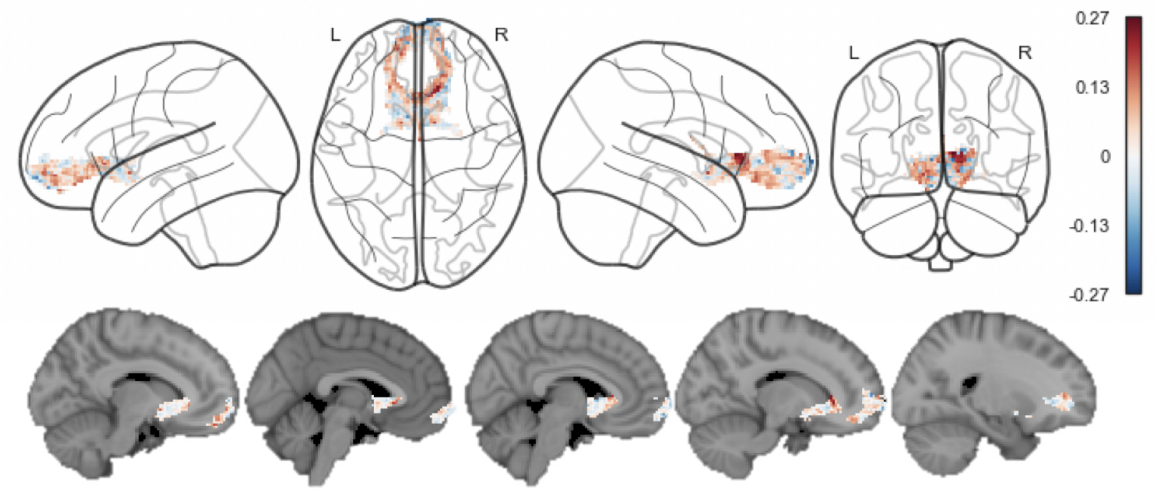

Fig. 2. Multivariate ridge regression weight maps showing where voxel coefficients either positively or negatively contributed to the prediction of self-esteem in the original data. These weight maps were then applied to the new sample to (a) The weight map trained on non-frontostriatal white matter voxels. (b) The weight map based on the frontostriatal voxels only. Note the higher weight values indicated in the legend in the frontostriatal tracts relative to the non-frontostriatal tracts.

white matter tracts.

Frontostriatal systems, including those linking the MPFC to the ventral striatum, have been linked to a variety of motivation and reward-related processes in both humans (Galvan et al., 2005) and non-human animals (Berridge \& Kringelbach, 2008). Researchers have also demonstrated that abnormalities in these systems are linked with the reductions in hedonic capacity that characterize disorders such major depression (Heller et al., 2009). Moreover, large longitudinal studies have also suggested that self-esteem is a causal factor in depression, such that low self-esteem increases vulnerability to depression rather than depression eroding self-esteem (Sowislo \& Orth, 2013). The current study provides further evidence that frontostriatal structural integrity may be a component of the biological basis for variability in self-esteem and may contribute to our understanding of the etiology of the link between self-esteem and depressive symptomology.

Standard methodological approaches in psychology have used traditional within-sample statistical analysis procedures to explain the relationship between measures of interest. Within this framework, one can estimate the relationship between two variables-like self-esteem and white matter integrity - to be able to explain the relationship between those measures in each sample. However, the utility of this explanation-focused framework has been challenged by broader trends in machine learning applications and forecasting methods which evaluate the strength of the inferences of a model by how well they predict data in an independent sample of data (Yarkoni \& Westfall, 2017). From this predictionfocused perspective, demonstrating a relationship between a psychological phenomenon and biological measure is only meaningful if a model of this relationship can be evaluated independently in an out-of-sample dataset. In the current study, we successfully applied the prediction framework in order to directly and quantitatively generalize the original findings to a new sample of participants. Moreover, there was a stronger association between the predictions based on the multivariate model than those of the raw correlation values between self-esteem and frontostriatal white matter integrity estimated in the new sample alone. This suggests that out-of-sample predictive approaches may be more sensitive in detecting the presence of an effect than direct replications with these kinds of data. This may provide more generalizable interpretations but also yield interpretable results undetected by standard explanation-based methods that do not include model-based prediction.

Although we show that these models significantly predicted self-esteem in the new sample, there are several details 
worth noting. First, the strength of these models to accurately predict self-esteem scores in the new sample is modest $(\mathrm{r}=$ .24). However, the predicted values found in the current study are on par with findings showing that a person's self-esteem is similarly related to peer ratings of self-esteem $(r=.20)$, and is well above strangers ratings of one's self-esteem $(r=-.06)$ shown in previous work (Vazire, 2010). Thus, we can say that our model of self-esteem based on frontostriatal white matter integrity can capture a similar degree of variability in a person's self-esteem score as a friend's rating of their selfesteem. Moreover, it is also possible that differences in average self-esteem scores and the dMRI acquisition and analysis protocols between the original sample and the new sample could have influenced predictive accuracy in a variety of ways. This is also true of the participant demographic information which was different across the two samples. This variability in scanner acquisition and analysis along with differences in participant demographics speak to the robustness of the effects found in the current study. That said, the success of the predictive utility of these models to other datasets may still be subject to the degree to which other samples are similar to the ones used here. To this end, we have provided the fitted model weight maps used in this study available via the Open Science Framework to researchers who wish to apply these models to their own samples using these measures. It is also worth noting that there was a mean difference in selfesteem scores between the two samples. Although this will not affect the predicted $\mathrm{R} 2$ values in the testing data, it can lead to increases in RMSE which are indicative of greater error in the models. Some predictive modeling approaches will standardize these outcomes before fitting the model to keep the R2 variables the same while reducing the RMSE. Even though the RMSE is greater in our analyses, we chose not to use this approach in this case because the predicted values would be in the same units as the self-esteem scale and easier to interpret the success of the predicted values from our analyses.

A strength of the current study is the use of the roundrobin design to capture real-world social relationship processing in brain-to-brain similarity patterns between closeknit individuals. Typical neuroimaging studies of person knowledge either use multiple social target stimuli that are highly recognizable and consistent across participants, such as political figures (Kelley et al., 2002) and celebrities (Thornton \& Mitchell, 2017), but interpersonally unknown to the participants themselves. Other studies use more intimate relationships, such as a participant's best friend or family member (Chavez et al., 2017), but targets remain idiosyncratic across participants. The round-robin design employed here captures the strengths of both approaches by allowing for well-known targets that are consistent across all members of a group. Furthermore, having multiple targets within each group allowed us to test brain-to-brain multivoxel similarity patterns among all pairwise members of each group and relate them to social relationship metrics in a way not possible in other designs with fictional characters or single targets.

In conclusion, the results of the current study provide further evidence that self-esteem is related to the connectivity between brain regions involved in self-referential processing and reward. We demonstrate this using an out-of-sample predictive modeling approach which allows us to quantitatively generalize these inferences beyond a single sample of participants. These results can be verified within our openly shared data, and the multivariate weight maps generated from this study are also openly shared and can be applied to any future predictive modeling analysis on the relationship between white matter integrity and self-esteem. Thus, the generalizability approach used here simultaneously provides more confidence in the original findings while also providing a direct, quantitative prediction that can be further examined in more heterogeneous samples of people.

\section{ACKNOWLEDGEMENTS}

Research funded by University of Oregon.

\section{OPEN SCIENCE PRACTICES}

Data and analysis code and materials used in this study are available at https://osf.io/hbq26/. This includes the deidentified dMRI fractional anisotropy data, self-esteem scores, frontostriatal and whole-brain region of interest masks used for each analysis, $R$ code for testing the univariate models, and Python code used for fitting and testing multivariate models and generating the weight maps from these analyses. Moreover, we have also included the multivariate weight maps generated from this study that can be directly applied to self-esteem predictions in any future study in the same manner as they were here.

\section{References}

Abraham, A., Pedregosa, F., Eickenberg, M., Gervais, P., Mueller, A., Kossaifi, J., Gramfort, A., Thirion, B., \& Varoquaux, G. (2014). Machine learning for neuroimaging with scikit-learn. Frontiers in Neuroinformatics, 8. https://doi.org/10.3389/fninf.2014.00014

Behrens, T. E. J., Berg, H. J., Jbabdi, S., Rushworth, M. F. S., \& Woolrich, M. W. (2007). Probabilistic diffusion tractography with multiple fibre orientations: What can we gain? NeuroImage, 34(1), 144-155. https://doi.org/10.1016/j.neuroimage.2006.09.018

Behrens, T. E. J., Woolrich, M. W., Jenkinson, M., JohansenBerg, H., Nunes, R. G., Clare, S., Matthews, P. M., Brady, J. M., \& Smith, S. M. (2003). Characterization and propagation of uncertainty in diffusion-weighted MR imaging. Magnetic Resonance in Medicine, 50(5), 1077-1088. https://doi.org/10.1002/mrm.10609

Berridge, K. C., \& Kringelbach, M. L. (2008). Affective neuroscience of pleasure: Reward in humans and animals. Psychopharmacology, 199(3), 457-480. https://doi.org/10.1007/s00213-008-1099-6

Chavez, R. S., \& Heatherton, T. F. (2015). Multimodal frontostriatal connectivity underlies individual differences in self-esteem. Social Cognitive and Affective Neuroscience, 10(3), 364-370. https://doi.org/10.1093/scan/nsu063

Chavez, R. S., \& Heatherton, T. F. (2017). Structural integrity of frontostriatal connections predicts longitudinal changes in selfesteem. Social Neuroscience, 12(3), 280-286. https://doi.org/10.1080/17470919.2016.1164753

Denny, B. T., Kober, H., Wager, T. D., \& Ochsner, K. N. (2012). A meta-analysis of functional neuroimaging 
studies of self- and other judgments reveals a spatial gradient for mentalizing in medial prefrontal cortex. Journal of Cognitive Neuroscience, 24(8), 1742-1752. https://doi.org/10.1162/jocn ${ }_{a 0} 0233$

Fleming, J. S., \& Courtney, B. E. (1984). The dimensionality of self-esteem: II. Hierarchical facet model for revised measurement scales. Journal of Personality and Social Psychology, 46(2), 404-421. https://doi.org/10.1037/0022-3514.46.2.404

Galvan, A., Hare, T. A., Davidson, M., Spicer, J., Glover, G., \& Casey, B. J. (2005). The Role of Ventral Frontostriatal Circuitry in Reward-Based Learning in Humans. Journal of Neuroscience, 25(38), 8650-8656. https://doi.org/10.1523/JNEUROSCI.2431-05.2005

Graham, M. S., Drobnjak, I., \& Zhang, H. (2016). Realistic simulation of artefacts in diffusion MRI for validating post-processing correction techniques. NeuroImage, 125, 1079-1094. https://doi.org/10.1016/j.neuroimage.2015.11.006

Groenewegen, H. J., Wright, C. I., Beijer, A. V. J., \& Voorn, P. (1999). Convergence and Segregation of Ventral Striatal Inputs and Outputs. Annals of the New York Academy of Sciences, 877(1), 49-63. https://doi.org/10.1111/j.1749-6632.1999.tb09260.x

Haxby, J. V., Connolly, A. C., \& Guntupalli, J. S. (2014). Decoding neural representational spaces using multivariate pattern analysis. Annual Review of Neuroscience, 37, 435-456. https://doi.org/10.1146/annurev-neuro062012-170325

Heller, A. S., Johnstone, T., Shackman, A. J., Light, S. N., Peterson, M. J., Kolden, G. G., Kalin, N. H., \& Davidson, R. J. (2009). Reduced capacity to sustain positive emotion in major depression reflects diminished maintenance of frontostriatal brain activation. Proceedings of the National Academy of Sciences, 106(52), 22445-22450. https://doi.org/10.1073/pnas.0910651106

Knutson, B., Adams, C. M., Fong, G. W., \& Hommer, D. (2001). Anticipation of increasing monetary reward selectively recruits nucleus accumbens. The Journal of Neuroscience, 21(16), RC159.

Miller, E. K., \& Cohen, J. D. (2001). An Integrative Theory of Prefrontal Cortex Function. Annual Review of Neuroscience, 24(1), 167-202. https://doi.org/10.1146/annurev.neuro.24.1.167

Orth, U., Robins, R. W., \& Widaman, K. F. (2012). Life-span development of self-esteem and its effects on important life outcomes. In Journal of Personality and Social Psychology (Vol. 102).

Patenaude, B., Smith, S. M., Kennedy, D. N., \& Jenkinson, M. (2011). A Bayesian model of shape and appearance for subcortical brain segmentation. NeuroImage, 56(3), 907-922. https://doi.org/10.1016/j.neuroimage.2011.02.046

Patil, P., Peng, R. D., \& Leek, J. T. (2016). What Should Researchers Expect When They Replicate Studies? A Statistical View of Replicability in Psychological Science.
Perspectives on Psychological Science, 11(4), 539-544. https://doi.org/10.1177/1745691616646366

Pedregosa, F., Varoquaux, G., Gramfort, A., Michel, V., Thirion, B., Grisel, O., Blondel, M., Prettenhofer, P., Weiss, R., Dubourg, V., Vanderplas, J., Passos, A., Cournapeau, D., Brucher, M., Perrot, M., \& Duchesnay, É. (2011). Scikit-learn: Machine Learning in Python. Journal of Machine Learning Research, 12(85), 2825-2830.

Sesack, S. R., Deutch, A. Y., Roth, R. H., \& Bunney, B. S. (1989). Topographical organization of the efferent projections of the medial prefrontal cortex in the rat: An anterograde tract-tracing study with Phaseolus vulgaris leucoagglutinin. Journal of Comparative Neurology, 290(2), 213-242. https://doi.org/10.1002/cne.902900205

Smith, S. M., Jenkinson, M., Johansen-Berg, H., Rueckert, D., Nichols, T. E., Mackay, C. E., Watkins, K. E., Ciccarelli, O., Cader, M. Z., Matthews, P. M., \& Behrens, T. E. J. (2006). Tract-based spatial statistics: Voxelwise analysis of multi-subject diffusion data. NeuroImage, 31(4), 1487-1505. https://doi.org/10.1016/j.neuroimage.2006.02.024

Song, S.-K., Sun, S.-W., Ramsbottom, M. J., Chang, C., Russell, J., \& Cross, A. H. (2002). Dysmyelination Revealed through MRI as Increased Radial (but Unchanged Axial) Diffusion of Water. NeuroImage, 17(3), 1429-1436. https://doi.org/10.1006/nimg.2002.1267

Sowislo, J. F., \& Orth, U. (2013). Does low self-esteem predict depression and anxiety? A meta-analysis of longitudinal studies. Psychological Bulletin, 139(1), 213-240. https://doi.org/10.1037/a0028931

Vazire, S. (2010). Who knows what about a person? The selfother knowledge asymmetry (SOKA) model. Journal of Personality and Social Psychology, 98(2), 281-300. https://doi.org/10.1037/a0017908

Wagner, D. D., Chavez, R. S., \& Broom, T. W. (2019). Decoding the neural representation of self and person knowledge with multivariate pattern analysis and data-driven approaches. Wiley Interdisciplinary Reviews: Cognitive Science, 10(1), e1482. https://doi.org/10.1002/wcs. 1482

Wagner, D. D., Haxby, J. V., \& Heatherton, T. F. (2012). The Representation of Self and Person Knowledge in the Medial Prefrontal Cortex. Wiley Interdisciplinary Reviews: Cognitive Science, 3(4), 451-470. https://doi.org/10.1002/wcs.1183

Weaverdyck, M. E., Lieberman, M. D., \& Parkinson, C. (2020). Tools of the Trade Multivoxel pattern analysis in fMRI: A practical introduction for social and affective neuroscientists. Social Cognitive and Affective Neuroscience, 15(4), 487-509. https://doi.org/10.1093/scan/nsaa057

Woo, C.-W., Chang, L. J., Lindquist, M. A., \& Wager, T. D. (2017). Building better biomarkers: Brain models in translational neuroimaging. Nature Neuroscience, 20(3), 365-377. https://doi.org/10.1038/nn.4478 
Yarkoni, T., \& Westfall, J. (2017). Choosing Prediction Over Explanation in Psychology: Lessons From Machine Learning: Perspectives on Psychological Science. https://doi.org/10.1177/1745691617693393 